\title{
An art gallery approach to ensuring that landmarks are distinguishable
}

\author{
Lawrence H. Erickson and Steven M. LaValle \\ Department of Computer Science \\ University of Illinois at Urbana-Champaign \\ \{lericks4, lavalle\}@uiuc.edu
}

\begin{abstract}
How many different classes of partially distinguishable landmarks are needed to ensure that a robot can always see a landmark without simultaneously seeing two of the same class? To study this, we introduce the chromatic art gallery problem. A guard set $S \subset P$ is a set of points in a polygon $P$ such that for all $p \in P$, there exists an $s \in S$ such that $s$ and $p$ are mutually visible. Suppose that two members of a finite guard set $S \subset P$ must be given different colors if their visible regions overlap. What is the minimum number of colors required to color any guard set (not necessarily a minimal guard set) of a polygon $P$ ? We call this number, $\chi_{G}(P)$, the chromatic guard number of $P$. We believe this problem has never been examined before, and it has potential applications to robotics, surveillance, sensor networks, and other areas. We show that for any spiral polygon $P_{s p i}, \chi_{G}\left(P_{s p i}\right) \leq 2$, and for any staircase polygon (strictly monotone orthogonal polygon) $P_{\text {sta }}, \chi_{G}\left(P_{\text {sta }}\right) \leq 3$. For lower bounds, we construct a polygon with $4 k$ vertices that requires $k$ colors. We also show that for any positive integer $k$, there exists a monotone polygon $M_{k}$ with $3 k^{2}$ vertices such that $\chi_{G}\left(M_{k}\right) \geq k$, and for any odd integer $k$, there exists an orthogonal polygon $R_{k}$ with $4 k^{2}+10 k+10$ vertices such that $\chi_{G}\left(R_{k}\right) \geq k$.
\end{abstract}

\section{INTRODUCTION}

Suppose a robot is navigating a region populated with colored landmarks. The robot is equipped with the following primitives: drive toward the landmark, drive away from the landmark, and drive in circles around the landmark. If this robot were in an area where two landmarks with the same color are visible, then its motion primitives may become unpredictable. If it can see two different green landmarks, then what is it to do when told "drive toward the green landmark"? This raises a natural question: How many classes of partially distinguishable guards are required to guard a given area (see Figure 11? Equivalently, how many classes of landmarks are required so that the robot can always see a landmark (so that it can always navigate), but never two landmarks of the same class (so that it does not get confused)? In this paper, we try to answer this question for bounded simply connected polygonal areas. We assume that a robot cannot see a given landmark if the polygon boundary is in the way.

There are many reasons why one would want to minimize the number of landmark classes. Adding more classes of landmarks means that a more sophisticated sensing system is required. An eight color camera is easier to construct than a 32-bit color camera. Even if a camera can see thousands or millions of colors, differences in light or shade could still make classification difficult. This was demonstrated in [12], in which more powerful cameras (in terms of number of
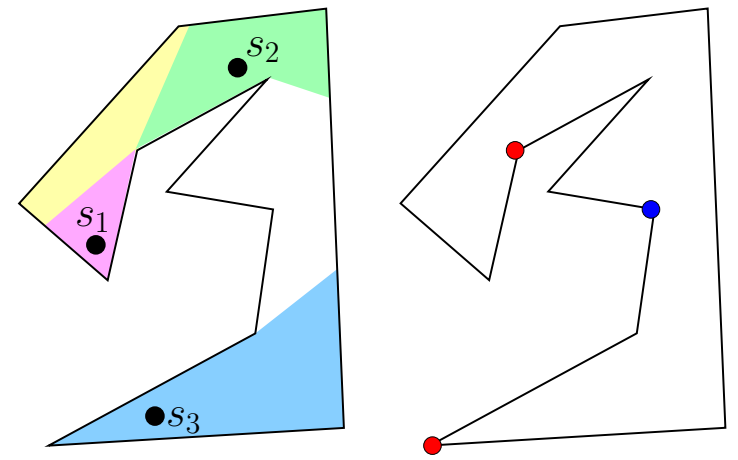

Fig. 1. [left] Three guards in a polygon $P$. The yellow region denotes the points that are visible from both $s_{1}$ and $s_{2}$, so $s_{1}$ and $s_{2}$ must be given different colors. The purple region is the area visible only from $s_{1}$, and the green region is the area visible only from $s_{2}$. The blue region is visible from $s_{3}$. Since this region does not intersect with the points visible from $s_{1}$ or $s_{2}$, the guard $s_{3}$ may be colored the same as $s_{1}$ or $s_{2}$. [right] A guard placement and coloring that uses only two colors. This is the minimum number of colors required for this polygon.

colors) were found to be worse for human iris identification than weaker cameras, as the more powerful cameras would see false differences in different pictures of the same eye. Minimizing the number of landmark classes could also make it so that only the "most different" classes are used, increasing the separation between sensor data points and decreasing classification errors. The problem of discovering the most distinctive visual landmarks for mobile robot navigation was addressed in [11]. Other research in which landmarks are specifically selected to reduce classification error include [20] and [24].

This is closely related to the original art gallery problem. It is impossible to list all of the significant results about art galleries in general polygons, but some of the most important works include results on tight bounds [2], [4] and exterior visibility [5]. Orthogonal art galleries are one of the most commonly studied variants, with notable results including tight bounds on the number of required guards [8], [15], [21] and bounds on the number of guards required for exterior visibility problems [7]. Results specific to monotone polygons include bounds on edge guards [1] and approximation algorithms with bounds independent of the number of polygon vertices [18]. Most of the important results from before 1987 are discussed in [22]. We prove lower bounds on the chromatic art gallery number for general, monotone, and orthogonal polygons.

We also prove upper bounds on the chromatic art gallery 
number for spiral polygons and staircase polygons (also known as strictly monotone orthogonal polygons). Spiral polygons are a heavily studied area in visibility. Special results for this class of polygons are available for the watchman route problem [19], the weakly cooperative guard problem [14], the visibility graph recognition problem [3], point visibility isomorphisms [16], and triangulation [25]. However, we are most interested in spiral polygons because of their use as building blocks. An algorithm for decomposing general polygons into a minimum number of spiral polygons was described in [10]. We choose to focus on spiral polygons because we think they could be a useful component in solving the chromatic guard number problem for general polygons, and staircase polygons for their similar potential as pieces of orthogonal polygons.

Section [II contains the formal definition of the problem. Section [II contains proofs for lower bounds on the chromatic guard number for general polygons, monotone polygons, and orthogonal polygons. Section IV contains upper bounds on the chromatic guard number for spiral polygons and staircase polygons. Section $\nabla$ discusses directions of future research.

\section{Problem Definition}

Let a polygon $P$ be a closed, simply connected, polygonal subset of $\mathbb{R}^{2}$ with boundary $\partial P$. A point $p \in P$ is visible from point $q \in P$ if the closed segment $\overline{p q}$ is a subset of $P$. The visibility polygon $V(p)$ of a point $p \in P$ is defined as $V(p)=\{q \in P \mid q$ is visible from $p\}$. Let a guard set $S$ be a finite set of points in $P$ such that $\bigcup_{s \in S} V(s)=P$. The members of a guard set are referred to as guards. A pair of guards $s, t \in S$ is called conflicting if $V(s) \cap V(t) \neq$ $\emptyset$. Let $C(S)$ be the minimum number of colors required to color a guard set $S$ such that no two conflicting guards are assigned the same color. Let $T(P)$ be the set of all guard sets of $P$. Let $\chi_{G}(P)=\min _{S \in T(P)} C(S)$. We call this value $\chi_{G}(P)$ the chromatic guard number of the polygon $P$. Note that the number of guards used can be as high or low as is convenient. We want to minimize the number of colors used, not the number of guards.

The notion of conflict can be phrased in terms of link distance. The link distance between two points $p, q \in P$ (denoted $L D(p, q)$ ) is the minimum number of line segments required to connect $p$ and $q$ via a polygonal path. Each line segment must be a subset of $P$.

Theorem 1. Two guards $s_{1}, s_{2} \in P$ conflict if and only if $L D\left(s_{1}, s_{2}\right) \leq 2$.

Proof: If $L D\left(s_{1}, s_{2}\right)=1$, then $s_{1}$ and $s_{2}$ are mutually visible, and obviously conflict.

If $L D\left(s_{1}, s_{2}\right)=2$, then there exists a point $r \in P$, such that $\overline{s_{1} r}, \overline{r s_{2}} \subseteq P$. Since $\overline{s_{1} r} \subseteq P, r \in V\left(s_{1}\right)$. Since $\overline{r s_{2}} \subseteq P$, $r \in V\left(s_{2}\right)$. Because $r$ is in $V\left(s_{1}\right)$ and $V\left(s_{2}\right)$, the intersection of $V\left(s_{1}\right)$ and $V\left(s_{2}\right)$ is non-empty; therefore $s_{1}$ and $s_{2}$ conflict.

If $s_{1}$ and $s_{2}$ conflict, then let $r$ be a point in the intersection of $V\left(s_{1}\right)$ and $V\left(s_{2}\right)$. Since $r \in V\left(s_{1}\right), \overline{s_{1} r} \subseteq P$. Since $r \in$ $V\left(s_{2}\right), \overline{r s_{2}} \subseteq P$. Because $\overline{s_{1} r}, \overline{r s_{2}} \subseteq P, L D\left(s_{1}, s_{2}\right) \leq 2$.

\section{LOWER BOUNDS ON THE CHROMATIC GUARD NUMBER}

A finite set of lines in the plane is a simple arrangement if each pair of lines intersects and no three lines intersect at the same point. A simple arrangement of lines can be used to construct a polygon that requires a linear number of colors relative to the number of vertices in the polygon.

Theorem 2. For every integer $k \geq 3$, there exists a polygon $P_{k}$ with $4 k$ vertices such that $\chi_{G}\left(P_{k}\right) \geq k$.

Proof: The polygon $P_{k}$ will be constructed from $k$ gadgets, each consisting of four line segments. Each gadget consists of a nearly triangular well and a line that connects to the next gadget. The goal is to arrange $k$ of these gadgets so that every pair of guards conflict, and each guard can guard no more than two convex vertices.

Let $T$ be a simple arrangement of $k$ lines. Now, make a closed convex $k$-gon bounding box $B$ that contains each intersection among the lines of $T$ in its interior, and has a boundary vertex on each line of $T$. Place the well of a very thin gadget at each of the boundary vertices (see Figure 2). Let $p_{1}$ and $p_{2}$ be two convex vertices in the same well associated with line $T_{1}$. Note that, as the opening of the well is made smaller, and the width of the segment joining $p_{1}$ and $p_{2}$ is made narrower, the distance between a point $q \in V\left(p_{1}\right) \cap B$ and the closest point to $q$ in $T_{1} \cap B$ becomes arbitrarily small. Note also that any guard placed in the well must lie on a line segment $\ell \subset V\left(p_{1}\right) \cup V\left(p_{2}\right)$ that extends from $\overline{p_{1} p_{2}}$ to a polygon edge connecting two reflex vertices on the other side of $P_{k}$.

Since each guard in a well has an $\ell$ segment that is arbitrarily close to its line from the arrangement, and all the lines in the arrangement intersect, the $\ell$ segments from two guards in different wells must intersect (assuming that the wells are thin and the well openings are narrow enough), so two guards in different wells must conflict. A guard $s$ located in $B$ must conflict with every guard, as every $\ell$ segment intersects $B$, and $B \subset V(s)$. Therefore, all guards placed in $P_{k}$ will pairwise conflict. Since $P_{k}$ has $2 k$ convex vertices, and each guard can see at most two convex vertices, $k$ guards are required; hence $\chi_{G}\left(P_{k}\right) \geq k$. Since $P_{k}$ is made from $k$ gadgets, each of which has four edges, $P_{k}$ has $4 k$ vertices.

A polygon $P$ is monotone if there exists a line $L$ such that the intersection of $P$ and any line perpendicular to $L$ has at most one connected component. A polygon $P$ is strictly monotone if there exists a line $L$ such that any line perpendicular to $L$ intersects $\partial P$ at two or fewer points.

Theorem 3. For every integer $k \geq 3$, there exists a strictly monotone polygon $M_{k}$ with $3 k^{2}$ vertices such that $\chi_{G}\left(M_{k}\right) \geq$ $k$.

Proof: The polygon $M_{k}$ is a variant of the standard "comb" used to show the occasional necessity of $\lfloor n / 3\rfloor$ guards in the standard art gallery problem [2]. The vertex list of $M_{k}$ is $[(1,2 k-2),(2,2 k-3),(4,2 k-3),(5,2 k-2),(6,2 k-$ 

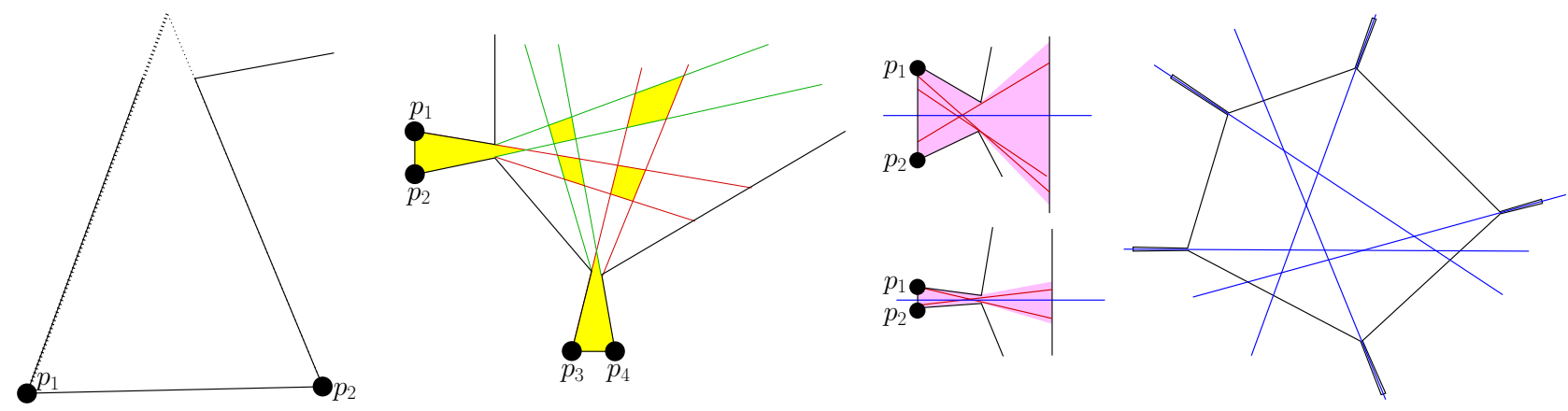

Fig. 2. [left] A gadget. The points $p_{1}$ and $p_{2}$ are the convex vertices. For a guard to see $p_{1}$ and $p_{2}$ simultaneously, it would have to be placed in the triangular region (bounded on top by the dotted lines) that does not extend far out of the well. [middle left] Two gadgets. The cones show the region outside of the well where a convex vertex is visible. The yellow regions are where a single guard can see two convex vertices. There is no place where a guard can see three convex vertices. [middle right] As the well opening is made smaller and the well is made more narrow, $V\left(p_{1}\right) \cup V\left(p_{2}\right)$ (purple region) becomes more narrow and any $\ell$ line segments (colored in red) from a guard in the well must get closer to arrangement line $T_{1}$ (colored in blue). [right] A polygon $P_{k}$ for $k=5$. The blue lines represent a simple arrangement $T$ of $k=5$ lines. Each line in the arrangement is associated with the well of a gadget.

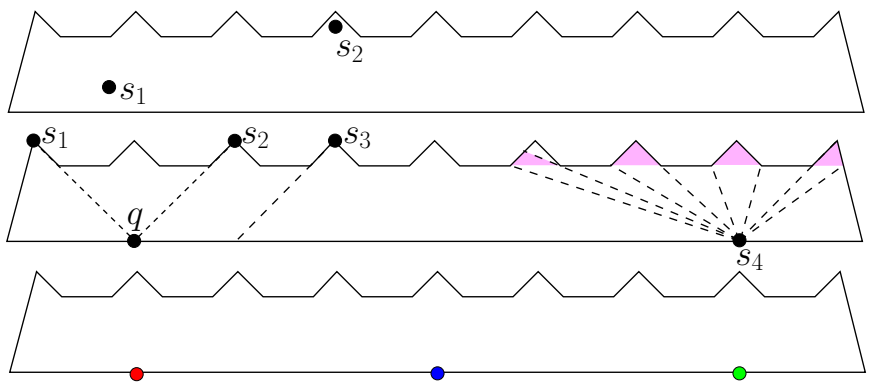

Fig. 3. [top] The polygon $M_{k}$ for $k=3$. The guard $s_{1}$ is a body guard, and the guard $s_{2}$ is an notch guard. [middle] Notch guards must be placed at least $k=3$ notches away from each other to avoid conflicts. Guards $s_{1}$ and $s_{2}$ conflict as $V\left(s_{1}\right) \cap V\left(s_{2}\right)=q$, but $s_{3}$, which is $k=3$ notches away from $s_{1}$ does not conflict with $s_{1}$. A body guard $s_{4}$ can only guard $k=3$ notches by itself. Portions of the rightmost four notches visible from $s_{4}$ are highlighted in purple. [bottom] A guard placement that requires three colors.

$\left.3) \ldots\left(4 k^{2}-4,2 k-3\right),\left(4 k^{2}-3,2 k-2\right),\left(4 k^{2}-2,0\right),(0,0)\right]$. This polygon has $3 k^{2}$ vertices, and it consists of a trapezoidal region (the body region) that has $k^{2}$ notches attached to the shorter edge. Call the vertices with a $y$ coordinate of $2 k-2$ apex points. Note that each notch has a unique apex point. A guard with coordinates $(x, y)$ will be referred to as a notch guard if $y>2 k-3$ and will be referred to as a body guard if $y \leq 2 k-3$ (see Figure 3).

Each body guard can guard up to $k$ distinct notches. However, since the visibility polygon of a body guard includes the entire body region, and every guard's visibility polygon intersects the body region, a body guard will conflict with every other guard in the polygon. Let $m_{b o d y}$ be the number of body guards used in a guard set of $M_{k}$.

Each notch guard can guard only one notch. However, two notch guards will not conflict if they are placed far enough away from each other. Since the bottom edge of $M_{k}$ has a $y$ coordinate of 0 , two notch guards are forced to conflict only if the distance between the apex points of their corresponding notches is $4 k-4$ or less. Let a set of $k$ notches be consecutive if the maximum distance between the apex points of any two notches in the set is $4 k-4$. Let $m_{n o t c h}$ be the maximum number of notch guards in any consecutive set of $k$ notches in $M_{k}$.
Suppose the polygon $M_{k}$ has a guard set $S$ assigned to it that requires only $\chi_{G}\left(M_{k}\right)$ colors. Consider $k$ consecutive notches in $M_{k}$ that contain $m_{n o t c h}$ notch guards in total. All of these notch guards will conflict with each other, and all of these notch guards will conflict with all of the body guards. Therefore, $\chi_{G}\left(M_{k}\right) \geq m_{\text {notch }}+m_{\text {body }}$. Now, note that each body guard can guard at most $k$ notches. Since there are $k^{2}$ notches, by the pigeonhole principle, notch guards can guard at most $\mathrm{km}_{\text {notch }}$ notches (see Figure 3). Since each notch must be guarded, $k m_{\text {notch }}+k m_{\text {body }} \geq k^{2}$, so $m_{\text {notch }}+m_{\text {body }} \geq k$. Therefore $\chi_{G}\left(M_{k}\right) \geq m_{\text {notch }}+m_{\text {body }} \geq k$.

A polygon $P$ is orthogonal (sometimes called rectilinear in other publications) if all of its angles are right angles.

Theorem 4. For every odd integer $k \geq 3$, there exists a monotone orthogonal polygon $R_{k}$ with $4 k^{\overline{2}}+10 k+10$ vertices such that $\chi_{G}\left(R_{k}\right) \geq k$.

Proof: We begin by introducing a family of orthogonal polygons with two parameters, $m, i \in \mathbb{Z}^{+}$. The vertex list for polygon $R_{m, i}$ is $[(0,0),(0, i+1),(1, i+1),(1, i),(2, i),(2, i+$ $1),(3, i+1),(3, i), \ldots,(2 m-2, i),(2 m-2, i+1),(2 m-1, i+$ $1),(2 m-1,0)]$. This takes the form of a $(2 m-1) \times i$ rectangle with $m 1 \times 1$-sized notches along the top edge (see Figure 4). Any guard in $R_{m, i}$ with a $y$-coordinate greater than $i$ will be called a notch guard. All other guards will be called body guards.

There are $m$ notches. Each notch has a ceiling of length 1. These ceilings are a subset of the polygon, so they must be covered. A body guard can cover the most ceiling if it is placed on the bottom of the polygon. Let $C(s)$ be the total length of ceiling that a body guard $s$ can see. Suppose a body guard $s$ is placed on the bottom of the polygon underneath the left edge of a notch (thus maximizing the amount of ceiling it can see to its right). This guard can see all of the notch that it is underneath. It can see a length of $(i-2) / i$ of the next notch to the right, $(i-4) / i$ of the notch after that, and so on (see Figure 4). Therefore, $s$ can see $\sum_{j=0}^{i / 2} 2 j / i$ ceiling to its right. We double this term to account for the ceiling it might be able to see on its left to get 


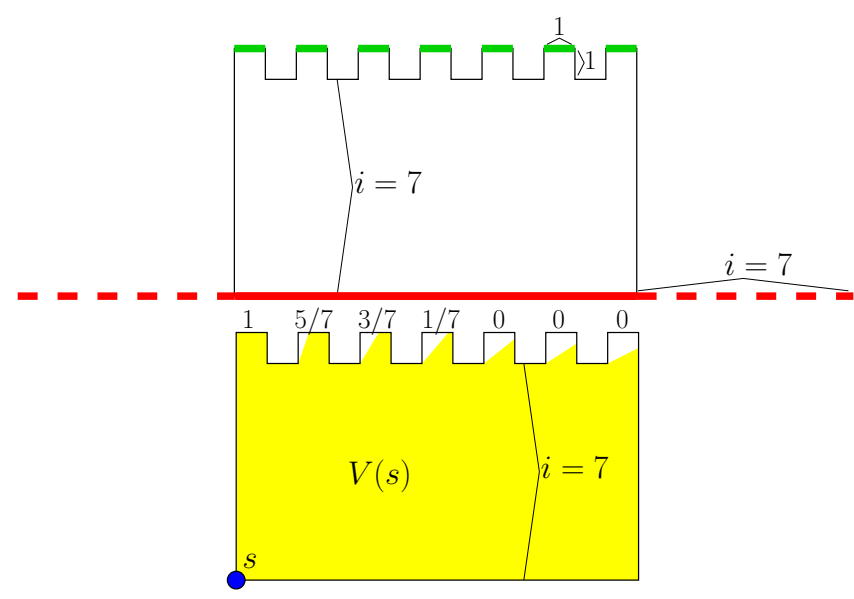

Fig. 4. [top] The polygon $R_{m, i}$ for $i=7$ and $m=7$. Each notch has a height and width of 1 . The bottom edge is highlighted in red, and the ceiling edges are highlighted in green. The dotted red line represents the extra length $i$ that we can assume exists on either side of the bottom edge for the purposes of placing nonconflicting notch guards. [bottom] A guard $s$ is placed on the bottom edge of the polygon is a position where the total length of ceiling edge in $V(s)$ to the right of $s$ is maximized. The visibility polygon $V(s)$ is highlighted in yellow. The number above each notch shows how much ceiling edge length in that notch is in $V(s)$.

$$
C(s) \leq \sum_{j=0}^{i / 2} \frac{4 j}{i}=\frac{4}{i}\left(\frac{\left(\frac{i}{2}\right)\left(\frac{i}{2}+1\right)}{2}\right)=\frac{i}{2}+1
$$

Suppose that a certain color is used instead for notch guards. Each notch guard can guard a ceiling of length 1 . However, while each body guard must have its own unique color, a single color can be assigned to multiple notch guards. So, given the dimensions of the polygon, how many notch guards can share one color? Note that the visibility polygon of a notch guard must include a portion of the bottom edge of the polygon. Since two notch guards that use the same color have visibility polygons that do not intersect, this space along the bottom edge of the polygon is a resource that can only support a finite number of notch guards of the same color. The bottom edge of the polygon has length $2 m-1$. However, to account for the fact that the bottom of the visibility polygons of notches close to the edge could have an additional length of up to $i$ if the convex portion of the polygon were wider, we can treat the bottom edge as though it has length $2 m+2 i-1$ (see Figure 5 ). It is clear that placing a notch guard $s$ along the ceiling of a notch minimizes the length of the bottom edge inside $V(s)$. It is also clear that for any point $p$ on the bottom edge of the polygon, there exists a point on the ceiling of a notch that is visible from $p$. Suppose a guard $s$ is placed on the ceiling of a notch at a length $0 \leq t \leq 1$ from the left vertex of the ceiling. Since the height of the notch is 1 , the leftmost point of $V(s)$ on the bottom edge will extend a distance $t i$ past the $x$-coordinate of the leftmost point in the notch. Similarly, the rightmost point of $V(s)$ will extend a distance of $(1-t) i$ past the $x$-coordinate of the rightmost point in the notch (see Figure 5). Therefore, the length of the bottom edge inside $V(s)$ is $i+1$ (we have to include the length of 1 directly underneath

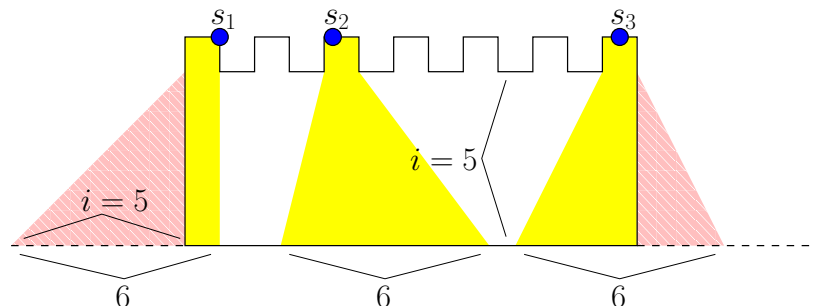

Fig. 5. The polygon $R_{m, i}$ for $i=5$ and $m=7$. Three guards have been placed on ceiling edges and their visibility polygons are highlighted in yellow. The striped pink regions are portions of the visibility polygons that have been cut off by the left or right side of $R_{m, i}$. Note that if the length of the bottom edge of $R_{m, i}$ extended an extra $i$ in both directions, then the length of the bottom edge of each visibility polygon would be $i+1=6$, regardless of the guard's location on its notch's ceiling.

the notch). This means that the amount of the bottom edge seen by a single notch guard placed on a ceiling is not related to its exact location within that ceiling. Since no two notch guards with the same color can have any of their visibility polygons overlap, a single color can be used to guard at most $(2 m+2 i-1) /(i+1)$ notches.

Choose any guard set for $R_{m, i}$. Let $x_{n o t c h}$ be the number of colors used in the notch guards, and let $x_{\text {body }}$ be the number of colors used in the body guards. Since each guard must be a notch or a body guard, we get

$$
x_{n o t c h}+x_{\text {body }}=\chi_{G}\left(R_{m, i}\right)
$$

Since each color used for a body guard can guard at most $i / 2+1$ length of ceiling, and each color used for notch guards can guard at most $(2 i+2 m-1) /(i+1)$ length of ceiling, and there is $m$ total length of ceiling, we get

$$
\left(\frac{2 i+2 m-1}{i+1}\right) x_{n o t c h}+\left(\frac{i}{2}+1\right) x_{b o d y} \geq m .
$$

Let $k=(i-3) / 2$ and let polygon $R_{k}$ be the polygon where $m=\left(i^{2}-i\right) / 4+1$ with $i \in\left\{x \in \mathbb{Z}^{+} \mid x \equiv 1 \bmod 4\right\}$. By the quadratic formula (and keeping in mind that $i$ must be positive), this implies that $i=1 / 2+\sqrt{4 m-(15 / 4)}$. This turns Equation 3 into

$$
\left(\frac{i}{2}+1\right)\left(x_{n o t c h}+x_{\text {body }}\right) \geq \frac{i^{2}-i}{4}+1 .
$$

The term $\left(i^{2}-i\right) / 4+1$ is equal to $\left(\left(i^{2}+2 i\right)-(3 i+6)+10\right) / 4$; hence Equation 4 can be rewritten as

$\chi_{G}\left(R_{k}\right)=x_{n o t c h}+x_{\text {body }} \geq \frac{i}{2}-\frac{3}{2}+\frac{10}{2 i+4} \geq \frac{i-3}{2}=k$.

The polygon therefore requires at least $(i-3) / 2=$ $\sqrt{m-(15 / 16)}-(5 / 4)$ colors. The polygon $R_{k}$ has $4 m$ vertices and $\chi_{G}\left(R_{k}\right) \geq \sqrt{m-(15 / 16)}-(5 / 4)$. Since $k=$ $(i-3) / 2=\sqrt{m-(15 / 16)}-(5 / 4), R_{k}$ has $4 k^{2}+10 k+10$ vertices and requires $k$ colors. The integer $k$ must be odd to ensure that the number of vertices is divisible by 4 . 
While these constructions do not work when the desired number of required colors is 1 or 2 , it is trivially easy to construct such polygons, as $\chi_{G}(P) \geq 1$ for all polygons, and $\chi_{G}(P) \geq 2$ for all non-star-shaped polygons.

\section{UPPER BOUNDS ON THE CHROMATIC GUARD NUMBER}

One could just give every guard its own color. Any polygon $P$ with $n$ vertices can be guarded by $\lfloor n / 3\rfloor$ guards (the art gallery theorem [2]), so $\chi_{G}(P) \leq\lfloor n / 3\rfloor$. However, this bound is unsatisfying, because colors can often be reused. There exist polygons with an arbitrarily high number of vertices that require only two colors. We prove bounds better than $\lfloor n / 3\rfloor$ for two categories of polygons.

\section{A. Spiral polygons}

A chain is a series of points $\left[p_{1}, p_{2}, \ldots, p_{n}\right]$ along with line segments connecting consecutive points. A subchain is a chain that forms part of the boundary of a polygon. The points $p_{1}$ and $p_{n}$ are called endpoints, and all other points are internal vertices. A convex subchain is a subchain where all the internal vertices have an internal angle of less than $\pi$ radians. A reflex subchain is a subchain where all the internal vertices have an internal angle of greater than $\pi$ radians. Note that convex and reflex subchains can trivially consist of a single line segment (if there are no internal vertices). A spiral polygon is a polygon with exactly one maximal reflex subchain (all reflex subchains of the spiral polygon must be contained within the maximal reflex subchain).

Theorem 5. For any spiral polygon $P, \chi_{G}(P) \leq 2$.

Proof: The spiral polygon consists of two subchains, a reflex subchain, and a convex subchain. Let $v_{s}$ and $v_{t}$ be the endpoints of the reflex subchain. Without loss of generality, assume that the path along the convex subchain from $v_{s}$ to $v_{t}$ runs clockwise. The guards will all be placed along the edges of the convex subchain.

Call the $n$th guard placed $s_{n}$. Place $s_{1}$ at $v_{s}$. Let $p_{n}$ be the point most clockwise along the convex subchain that is visible from $s_{n}$. Let $b_{n}$ be the most counterclockwise vertex along the reflex subchain visible from $s_{n}$. Let $g_{n}$ be the vertex immediately clockwise from $b_{n}$. Let $r_{n}$ be the point on the convex subchain colinear with $g_{n}$ and $b_{n}$ and visible from both. Note that $p_{n}$ and $r_{n}$ define the endpoints of an interval along the convex subchain. Place $s_{n+1}$ at a point on this interval that is not one of the endpoints. Note that this means $s_{n+1} \notin$ $V\left(s_{n}\right)$. Terminate when a guard can see $v_{t}$ (see Figure 6).

We can show that this is a guard set for the polygon by triangulating the polygon using the polygon vertices, the members of $S$, and the points $p_{i}$ and showing that each triangle has a member of $S$ as one of its vertices. Suppose that the polygon bounded by the edges starting from $p_{n}$ counterclockwise along the boundary of $P$ until $b_{n}$ and the edge between $p_{n}$ and $b_{n}$ has already been triangulated such that each triangle contains a vertex in the set $\left\{s_{i} \mid i \leq n\right\}$. We must show that $s_{n+1}$ can guard the subpolygon bordered by the edges counterclockwise from $p_{n+1}$ to $p_{n}$, the edge between

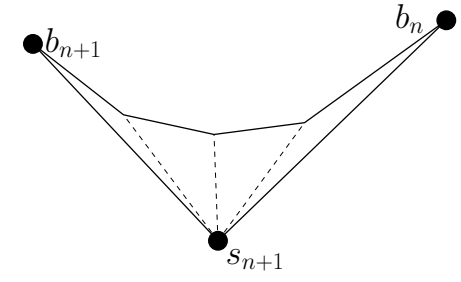

Fig. 7. A polygon consisting of the edges on the reflex subchain between $b_{n}$ and $b_{n+1}$ and the edges $\overline{s_{n+1} b_{n}}$ and $\overline{s_{n+1} b_{n+1}}$. Since all the vertices on the reflex subchain are reflex, this polygon has only one triangulation, where all triangles have $s_{n+1}$ as an endpoint.

$p_{n}$ and $b_{n}$, the vertices counterclockwise from $b_{n}$ to $b_{n+1}$, and the edge between $b_{n+1}$ and $p_{n+1}$ (call this subpolygon $P_{n+1}$ ). If each of these vertices in the subpolygon is visible from $s_{n+1}$, then the subpolygon can be triangulated by connecting each vertex to $s_{n+1}$, meaning that $s_{n+1}$ guards the entire subpolygon (see Figure 6).

Since $s_{n+1}$ is placed on the interval in between $p_{n}$ and $r_{n}$, it must be able to see the entire edge between $g_{n}$ and $b_{n}$, meaning that $b_{n}$ is visible from $s_{n+1}$. By definition, the vertex $b_{n+1}$ is visible from $s_{n+1}$. Examine the polygon consisting of the edges along the reflex subchain between $b_{n}$ and $b_{n+1}$, $\overline{s_{n+1} b_{n}}$, and $\overline{s_{n+1} b_{n+1}}$. Since all the vertices along the reflex subchain are reflex, they cannot have edges between each other in a triangulation, so in any triangulation, they must all be connected to $s_{n+1}$ (see Figure 7). By definition, the point $p_{n+1}$ is visible from $s_{n+1}$. The point $p_{n}$ is visible to $s_{n+1}$ because $s_{n+1}$ is on the convex subchain interval between $p_{n}$ and $r_{n}$. If two points on the convex subchain interval between $p_{n}$ and $r_{n}$ are not mutually visible, then there must be a reflex vertex between $b_{n}$ and $g_{n}$ on the reflex subchain, but by definition, there are no such vertices. Because the vertices in between $p_{n}$ and $p_{n+1}$ lie on a convex subchain, if $s_{n+1}$ can see both $p_{n}$ and $p_{n+1}$, then $s_{n+1}$ can see all the vertices in between. This means that $P_{n+1}$ can be triangulated with every triangle having $s_{n+1}$ as an endpoint, so $s_{n+1}$ guards $P_{n+1}$ (the triangle with endpoints $p_{n+1}, b_{n+1}$, and $s_{n+1}$ is degenerate, as those three points are colinear, but this is not a problem). This technique still works if $s_{n+1}$ can see $v_{t}$ (in this case, $p_{n+1}=b_{n+1}=v_{t}$ ). This implies inductively that $S$ is a guard set for $P$.

Because all the guards are along the convex subchain, if two guards conflict, their visibility polygons must intersect somewhere along the convex subchain. Also, since $s_{n} \notin V\left(s_{n+1}\right)$ and $s_{n} \notin V\left(s_{n-1}\right), s_{n+1}$ cannot conflict with $s_{n-1}$, or there would be no room along the convex subchain to place $s_{n}$. Therefore, all evenly indexed guards can be colored red, and all oddly indexed guards can be colored blue, so $\chi_{G}(P) \leq 2$.

\section{B. Staircase polygons}

An alternating subchain is a subchain with at least one internal vertex, with the first and last internal vertices being convex, and with consecutive internal vertices alternating between convex and reflex. A staircase polygon is an orthogonal polygon consisting of two convex vertices, $v_{w}$ and $v_{z}$, connected by two alternating subchains. For simplicity, we will 


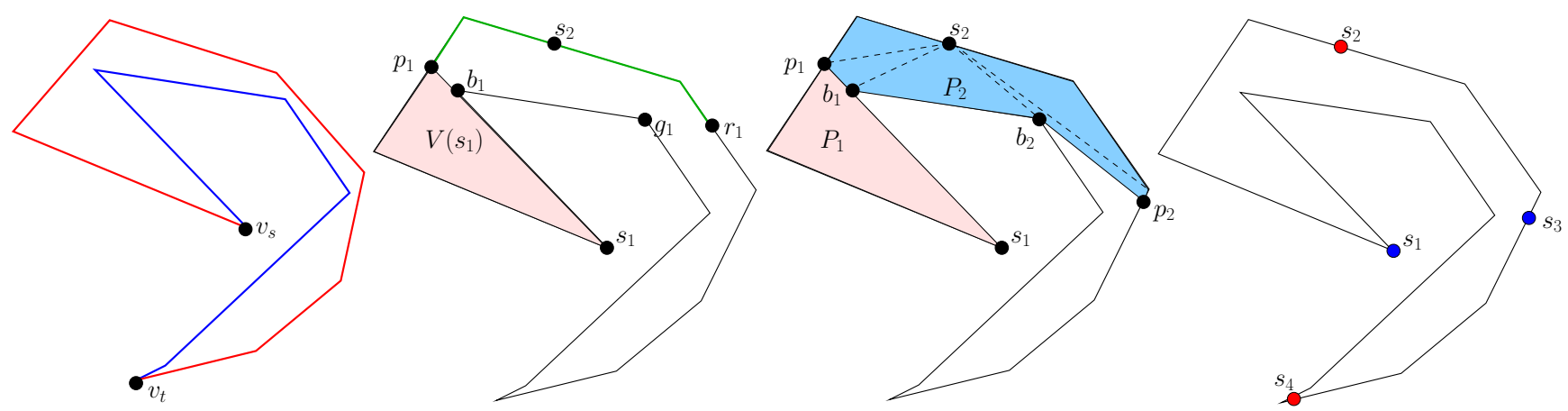

Fig. 6. [top left] A spiral polygon $P$. The convex subchain is highlighted in red, and the reflex subchain is highlighted in blue. [top right] The first guard $s_{1}$ is placed on vertex $v_{s}$. The points $p_{1}, b_{1}, g_{1}$, and $r_{1}$ are marked and the interval in which $s_{2}$ can be placed is highlighted in green. [bottom left] Recursively showing that placed guards form a guard set. The subpolygon $P_{1}$ is assumed to be guarded by $s_{1}$. The region that $s_{2}$ is responsible for is $P_{2}$, bounded by the reflex subchain between $b_{1}$ and $b_{2}$, the edge between $p_{2}$ and $b_{2}$, the convex subchain between $p_{2}$ and $p_{1}$, and the edge between $b_{1}$ and $p_{1}$. The subpolygon $P_{2}$ has been triangulated, indicating that $s_{2}$ can guard the whole subpolygon. The triangle with endpoints $p_{2}, b_{2}$, and $s_{2}$ is degenerate, as those three points are colinear. [bottom right] A guard placement and 2-coloring.

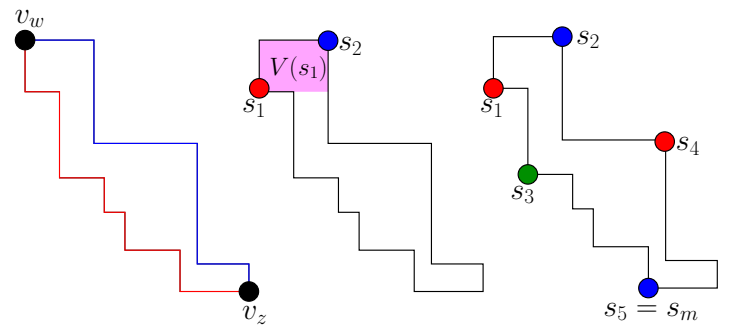

Fig. 8. [left] A staircase polygon $P$ with vertices $v_{w}$ and $v_{z}$ identified. The lower subchain is highlighted in red, and the upper subchain is highlighted in blue. [middle] The guard $s_{1}$ is placed on the neighbor of $v_{w}$ on the lower subchain. The guard $s_{2}$ is placed on the rightmost convex vertex in $V\left(s_{1}\right)$. [right] A guard placement and coloring for $P$ that uses only three colors.

assume without loss of generality that orthogonal polygons are always oriented such that each edge is either vertical or horizontal, and that $v_{w}$ is the top left vertex, and that $v_{z}$ is the bottom right vertex. Put the polygon on a coordinate plane with $v_{w}$ at the $(0,0)$ coordinate, let right be the positive $x$ direction, and let up be the positive $y$ direction. The term "staircase polygon" is a synonym for strictly monotone orthogonal polygon (mentioned in [7], which solved the prison yard problem for this class of polygons). Note that the bound from Theorem 4 is for monotone orthogonal polygons, not strictly monotone orthogonal polygons.

Theorem 6. For any staircase polygon $P, \chi_{G}(P) \leq 3$.

Proof: Due to our assumptions about the orientation of the polygon $P$, one of the alternating subchains is going to be above the other one. Call the higher subchain the upper subchain and call the other subchain the lower subchain. Place a guard $s_{1}$ on the neighbor of $v_{w}$ along the lower subchain. If guard $s_{i}$ has been placed on the lower subchain, then place guard $s_{i+1}$ on the right-most convex vertex on the upper subchain that is contained in $V\left(s_{i}\right)$. If guard $s_{i}$ has been placed on the upper subchain, then place guard $s_{i+1}$ on the right-most convex vertex on the lower subchain that is contained in $V\left(s_{i}\right)$. Stop placing guards when a guard can see $v_{z}$, and let $m$ be the number of guards placed (see Figure 8).

First, it must be shown that $s_{i}$ and $s_{i+2}$ are not placed on the same vertex. Suppose without loss of generality that $s_{i}$ is on the lower subchain. Note that the rightmost convex vertex on the lower subchain in $V\left(s_{i+1}\right)$ must also be the lowest convex vertex on the lower subchain in $V\left(s_{i+1}\right)$. Note also that a ray extended downward from $s_{i+1}$ must intersect the horizontal edge incident to $s_{i+2}$ (otherwise $s_{i+2}$ would not be the rightmost convex vertex on the lower subchain). If this is the same horizontal edge that is incident to $s_{i}$, then the point where the ray intersects the horizontal edge incident to $s_{i}$ must be a convex vertex (call it $v_{f}$ ). Since the convex vertex $v_{f}$ neighbors the convex vertex $v_{i}$ along a horizontal edge, and since $v_{f}$ is to the right of $v_{i}, v_{f}$ must be $v_{z}$. Therefore, $s_{i+2}$ would only be placed on the same vertex as $s_{i}$ if $v_{z}$ is visible from $s_{i+1}$. Since we stop placing guards once a guard can see $v_{z}$, two guards will never be placed on the same vertex.

Next, it must be shown that this is a guard set for the staircase polygon. Suppose without loss of generality that guard $s_{i}$ is placed on the lower subchain. Assume that the set $\left[s_{1}, s_{2} \ldots s_{i}\right]$ forms a guard set for the subpolygon that lies above the guard $s_{i}$ (call this subpolygon $P_{i}$ ). We must show that the set $\left[s_{1}, s_{2} \ldots s_{i+1}\right]$ forms a guard set for the subpolygon that lies to the left of guard $s_{i+1}$ (call this subpolygon $\left.P_{i+1}\right)$. Let $p_{i+1}$ be the point where a ray extended downward from $s_{i+1}$ intersects the lower subchain. Note that each vertex on the lower subchain between $s_{i}$ and $p_{i+1}$ is visible from $s_{i+1}$. We have to show that $s_{i+1}$ guards $P_{i+1} \backslash P_{i}$. Let $v_{i}^{r}$ be the reflex vertex to the right of $s_{i}$ on the lower subchain. Let $Q_{i+1}$ be the subpolygon below $s_{i+1}$ and to the left of $s_{i+1}$ (see Figure 9). Clearly, $Q_{i+1} \supseteq P_{i+1} \backslash P_{i}$ (as $s_{i+1}$ cannot be lower than $s_{i}$ ). Note that every vertex of $Q_{i+1}$ that is not connected to $s_{i+1}$ by an edge of $Q_{i+1}$ is on the lower subchain. For any given vertex $v$ in $Q_{i+1}$ that is not connected to $s_{i+1}$ by an edge of $Q_{i+1}$, all edges of $Q_{i+1}$ not incident to $s_{i+1}$ that lie above $v$ must also lie to the left of $v$, and all edges of $Q_{i+1}$ not incident to $s_{i+1}$ that lie to the right of $v$ must also lie below $v$. Since $s_{i+1}$ is never lower than $v$, and never to the right of $v$, every vertex $v$ of $Q_{i+1}$ must be visible from $s_{i+1}$. This means that one could triangulate $Q_{i+1}$ such that each triangle has $s_{i+1}$ as one of its corners. Therefore, 


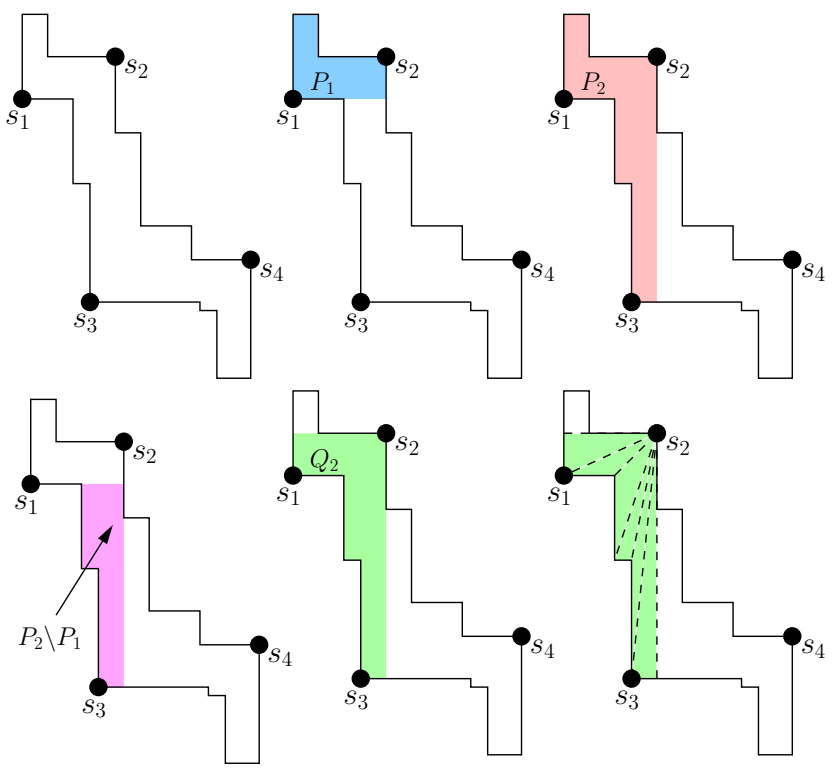

Fig. 9. [top left] A polygon $P$ with a guard placement. [top middle] The region $P_{1}$ that $s_{1}$ is responsible for guarding. [top right] The region $P_{2}$ that $s_{1}$ and $s_{2}$ are reponsible for guarding. [bottom left] The region $P_{2} \backslash P_{1}$ that $s_{2}$ is responsible for guarding. [bottom middle] The region $Q_{2}$, which consists of the portion of $P$ below and to the left of $s_{2}$. This region is a superset of $P_{2} \backslash P_{1}$. [bottom right] A triangulation of $Q_{2}$ where all triangles have a vertex at the location of $s_{2}$, showing that $s_{2}$ guards $Q_{2}$.

the guard $s_{i+1}$ can guard $Q_{i+1}$ by itself. Therefore, the set $\left[s_{1}, s_{2} \ldots s_{m}\right]$ forms a guard set for $P$.

Finally, it must be shown that the guard set $\left[s_{1}, s_{2} \ldots s_{m}\right]$ can be colored with three colors. Suppose guard $s_{i}$ is placed on the lower chain. Let $y_{i}$ be the $y$-coordinate of the lowest point visible from $s_{i}$. Note that, because $s_{i}$ is on a convex rightangle vertex on the lower subchain, $V\left(s_{i}\right)$ is bordered on the bottom by a horizontal line at the same height as the horizontal edge incident to $s_{i}$; therefore $y_{i}$ is just the $y$-coordinate of $s_{i}$. Let $y_{i+3}$ be the $y$ coordinate of the highest point in $V\left(s_{i+3}\right)$. Because $s_{i+3}$ is on a convex right-angle vertex on the upper subchain, $V\left(s_{i+3}\right)$ is bordered on top by a horizontal line at the same height as the horizontal edge incident to $s_{i+3}$; therefore $y_{i+3}$ is just the $y$-coordinate of $s_{i+3}$. Now, we must show that $y_{i}>y_{i+3}$. In the portion of the proof that showed that each guard is placed on a unique vertex, we demonstrated that the $y$-coordinate of $s_{i+1}$ (call it $y_{i+1}$ ) has to be higher than the $y$-coordinate of $s_{i+3}$. If $y_{i} \leq y_{i+3}$, then $y_{i} \leq y_{i+3}<y_{i+1}$. However, this is impossible, because $s_{i+1}$ was placed on the rightmost (and thus, lowest) vertex on the upper chain that was in $V\left(s_{i}\right)$. Therefore, $y_{i}>y_{i+3}$. Since the highest point in $V\left(s_{i+3}\right)$ is lower than the lowest point in $V\left(s_{i}\right), s_{i}$ and $s_{i+3}$ cannot conflict (see Figure 10).

Since $s_{i}$ and $s_{i+3}$ do not conflict, we can color all guards with an index of $0 \bmod 3$ with green, all guards with an index of $1 \bmod 3$ with red, and all guards with an index of $2 \bmod 3$ with blue. Therefore $\chi_{G}(P) \leq 3$.

We have assumed throughout this proof that guard $s_{i}$ was placed on the lower subchain. However, the arguments made above still apply if $s_{i}$ was placed on the upper subchain (reflect the polygon over the $y=-x$ line).

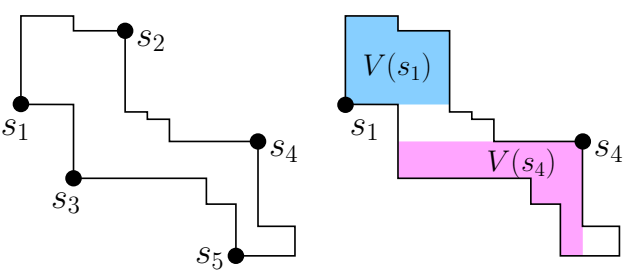

Fig. 10. [left] A staircase polygon $P$ with a guard placement. [right] The regions $V\left(s_{1}\right)$ and $V\left(s_{4}\right)$ are shown. Note that the lowest point in $V\left(s_{1}\right)$ is higher than the highest point in $V\left(s_{4}\right)$, as the horizontal line incident to $s_{1}$ 's vertex is higher than the horizontal line incident to $s_{4}$ 's vertex.

\section{CONCLusion}

We have introduced the chromatic art gallery problem, which asks for the minimum number of landmark colors required to ensure that a robot travelling in a given polygon can always see at least one landmark, but never simultaneously sees two of the same color. We have constructed a polygon with $n$ vertices that requires $\Omega(n)$ colors, and we have constructed monotone and orthogonal polygons that require $\Omega(\sqrt{n})$ colors. We have also found constant upper bounds on the chromatic guard number for the spiral and staircase polygons. These two families of polygons may be useful as building blocks for polygons in more general families.

The results from Section IIII seem to indicate that the environments that have the highest chromatic guard number have a large central convex region with several smaller niches attached to it. Therefore, if one were designing an environment where robots were to navigate via visual landmarks, it may be advantageous to design the environment without such a region, as that region would require more landmark classes and would potentially be more susceptible to classification errors.

Some directions of future research would be finding bounds for other families of polygons, and finding tight bounds for the general, monotone, and orthogonal polygons. Visibility in curvilinear bounded regions has also been researched [9]. Allowing polygons with holes is another possibility, as is placing further restrictions on the placement of guards, perhaps forcing the guards to be strongly cooperative [23] or weakly cooperative [17].

The problem could also be attacked from a visibility graph context. The structure of standard visibility graphs for general polygons is still not completely understood, but [6] gives four necessary conditions for visibility graphs. It is likely that analogues of these four conditions could be made for "2-link" visibility.

There are also algorithmic questions. While finding the minimum number of art gallery guards for a given polygon is NP-complete [13], it is not necessary to find the minimum number of art gallery guards to find the minimum number of colors required for a polygon (see Figure 11). There is also the possiblity that the graphs representing the conflict relationships between guards (each graph vertex is a guard, and two vertices are connected by an edge if the corresponding guards conflict) is an easy family of graphs to color. However, these graphs 

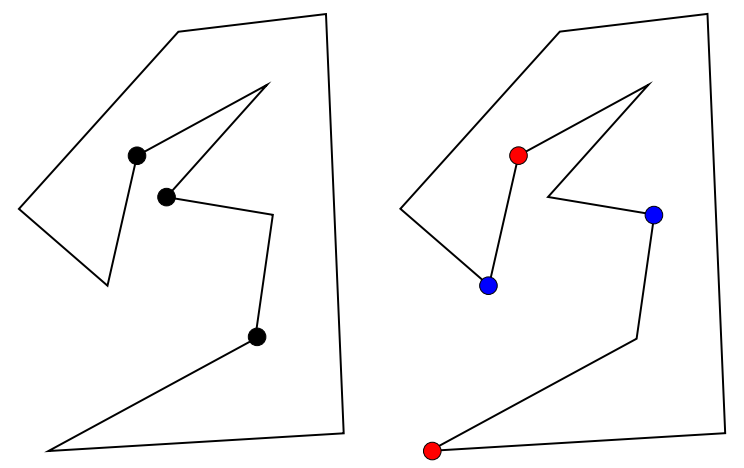

Fig. 11. [left] A polygon $P$ with a minimal guard set of three guards. [right] A guard placement and coloring for $P$ that uses the minimum two colors, but four guards. This demonstrates that a guard placement that uses the minimum number of colors does not need to use the minimum number of guards.

are not generally perfect graphs, and there are relatively few non-perfect families of graphs that are easy to color.

Finally, for practical robotics purposes, it would be useful to make a more realistic model of when guards conflict. For example, it may be interesting to research the case where the robot has limited vision, so that two guards sufficiently far from each other will not conflict even if there is no obstacle between them.

\section{Acknowledgments}

This work is supported in part by and NSF grant 0904501 (IIS Robotics), NSF grant 1035345 (CNS Cyberphysical Systems), DARPA SToMP grant HR0011-05-1-0008, and MURI/ONR grant N00014-09-1-1052.

\section{REFERENCES}

[1] I. Bjorling-Sachs and D. L. Souvaine. A tight bound for edge guards in monotone polygons. In Canadian Conference on Computational Geometry, 1992.

[2] V. Chvatal. A combinatorial theorem in plane geometry. Journal of Combinatorial Theory Series B, 18(1):39-41, 1975.

[3] H. Everett and D. G. Corneil. Recognizing visibility graphs of spiral polygons. Journal of Algorithms, 11(1): 1-26, 1990.

[4] S. Fisk. A short proof of Chvatal's watchman theorem. Journal of Combinatorial Theory Series B, 24(3):374, 1978.

[5] Z. Furedi and D. J. Kleitman. The prison yard problem. Combinatorica, 14(3):287-300, 1994.

[6] S. K. Ghosh. On recognizing and characterizing visibility graphs of simple polygons. Discrete and Computational Geometry, 17(2):143-162, 1997.

[7] F. Hoffman and K. Kriegel. A graph-coloring result and its consequences for polygon-guarding problems. SIAM Journal on Discrete Mathematics, 9(2):210-224, 1996.

[8] J. Kahn, M. Klawe, and D. Kleitman. Traditional galleries require fewer watchmen. SIAM Journal on Algebraic and Discrete Methods, 4(2):194-206, 1983.

[9] M. I. Karavelas, C. D. Toth, and E. P. Tsigaridas. Guarding curvilinear art galleries with vertex or point guards.
Computational Geometry: Theory and Applications, 42 (6-7):522-535, 2009.

[10] J. M. Keil. Decomposing a polygon into simpler components. SIAM Journal on Computing, 15(4):799-817, 1985.

[11] M. Knapek, R.S. Oropeza, and D. J. Kriegman. Selecting promising landmarks. In Proc. IEEE International Conference on Robotics and Automation, pages 3771-3777, 2000.

[12] Emine Krichen, Mohamed Chenafa, Sonia GarciaSalicetti, and Bernadette Dorizzi. Color-based iris verification. In Seong-Whan Lee and Stan Li, editors, Advances in Biometrics, volume 4642 of Lecture Notes in Computer Science, pages 997-1005. Springer Berlin / Heidelberg, 2007.

[13] D. T. Lee and A. K. Lin. Computational complexity of art gallery problems. IEEE Transactions on Information Theory, 32(2):276-282, 1986.

[14] B. C. Liaw and R.C.T. Lee. An optimal algorithm to solve the minimum weakly cooperative guards problem for 1-spiral polygons. Information Processing Letters, 52 (2):69-75, 1994.

[15] A. Lubiw. Decomposing polygonal regions into convex quadrilaterals. In Proc. Symposium on Computational Geometry, pages 97-106, 1985.

[16] G. MacDonald and T. Shemer. Isomorphism of spiral polygons. Discrete and Computational Geometry, 16: 277-304, 1996.

[17] T. S. Michael and V. Pinciu. Art gallery theorems for guarded guards. Computational Geometry: Theory and Applications, 26(3):247-258, 2003.

[18] B. Nilsson. Approximate guarding of monotone and rectilinear polygons, pages 1362-1373. Lecture Notes in Computer Science. Springer Berlin/Heidelberg, 2005.

[19] B. J. Nilsson and D. Wood. Optimum watchmen routes in spiral polygons. In Canadian Conference on Computational Geometry, 1990.

[20] C. F. Olson. Selecting landmarks for localization in natural terrain. Autonomous Robotics, 12(1):201-210, 2002.

[21] J. O'Rourke. An alternate proof of the rectilinear art gallery theorem. Journal of Geometry, 21(1):118-130, 1983.

[22] J. O'Rourke. Art Gallery Theorems and Algorithms. Oxford University Press, Cambridge, UK, 1987. ISBN 0-19-503965-3.

[23] V. Pinciu. A coloring algorithm for finding connected guards in art galleries, pages 257-263. Lecture Notes in Computer Science. Springer Berlin/Heidelberg, 2003.

[24] S. Thrun. Finding landmarks for mobile robot navigation. In Proc. IEEE International Conference on Robotics and Automation, pages 958-963, 1998.

[25] G. Toussaint. Efficient triangulation of simple polygons. The Visual Computer, 7(5-6):280-295, 1991. 